Теорія Ймовір. та Матем. Статист. Вип. 79, 2008
Theor. Probability and Math. Statist.

No. 79, 2009, Pages 179-185

S 0094-9000(09)00777-7

Article electronically published on December 30, 2009

\title{
THE CONVERGENCE OF GALTON-WATSON BRANCHING PROCESSES WITH IMMIGRATION TO A DIFFUSION PROCESS
}

UDC 519.21

\author{
YA. M. KHUSANBAEV
}

\begin{abstract}
A sequence of almost critical Galton-Watson branching processes with immigration is studied. Sufficient conditions for the weak convergence of such processes to a diffusion process are found.
\end{abstract}

Let, for all $n \in \mathbb{N},\left\{\xi_{k, j}^{(n)}, k, j \in \mathbb{N}\right\}$ and $\left\{\varepsilon_{k}^{(n)}, k \in \mathbb{N}\right\}$ be two sequences of independent nonnegative integer-valued and identically distributed random variables. We introduce the sequence of random variables $X_{k}^{(n)}, k \in \mathbb{N}$, defined by the following recursion relations:

$$
X_{0}^{(n)} \equiv 0, \quad X_{k}^{(n)}=\sum_{j=1}^{X_{k-1}^{(n)}} \xi_{k, j}^{(n)}+\varepsilon_{k}^{(n)}, \quad k, n \in \mathbb{N} .
$$

If one treats the random variable $\xi_{k, j}^{(n)}$ as the number of offsprings of a $j$-th member of a population in its $(k-1)$-th generation and $\varepsilon_{k}^{(n)}$ as the number of members of the population immigrating to the $k$-th generation, then $X_{k}^{(n)}$ is the total number of members of the population in its $k$-th generation. The stochastic processes described above are called the Galton-Watson branching processes with immigration. Assume that the first two moments

$$
m_{n}=\mathrm{E} \xi_{1,1}^{(n)}, \quad \lambda_{n}=\mathrm{E} \varepsilon_{1}^{(n)}, \quad \sigma_{n}^{2}=\operatorname{Var} \xi_{1,1}^{(n)}, \quad b_{n}^{2}=\operatorname{Var} \varepsilon_{1}^{(n)}
$$

are finite for all $n \in \mathbb{N}$. A stochastic process defined by (1) is called subcritical, critical, or supercritical if $m_{n}<1, m_{n}=1$, or $m_{n}>1$, respectively. Further, a process given by (10) is called almost critical if $m_{n} \rightarrow 1$ as $n \rightarrow \infty$.

Wei and Winnicki [1] consider the random step function

$$
X^{(n)}(t)=X_{[n t]}^{(n)}, \quad t \in \mathbb{R}_{+}, n \in \mathbb{N},
$$

for the case where the distributions of random variables $\xi_{i, j}^{(n)}$ and $\varepsilon_{1}^{(n)}$ do not depend on $n$ and $m=1$; that is, they study the critical case where $\lambda_{n}=\lambda, \sigma_{n}^{2}=\sigma^{2}, m_{n}=m$, and $b_{n}^{2}=b^{2}$. Wei and Winnicki [1] do not deal with the case of a scheme of series. Note that the trajectories of the random step function belong to the Skorokhod space $D\left(\mathbb{R}_{+}\right)$. Let $[\cdot]$ denote the integer part of a number and $\mathbb{R}_{+}=[0, \infty)$. Wei and Winnicki [1] proved the weak convergence

$$
\frac{1}{n} X^{(n)} \stackrel{D}{\rightarrow} X \quad \text { as } n \rightarrow \infty
$$

2000 Mathematics Subject Classification. Primary 60J80; Secondary 60F17, 60J60.

Key words and phrases. Almost critical Galton-Watson branching process with immigration, weak convergence, stochastic differential equation, Skorokhod's space. 
in the space $D\left(\mathbb{R}_{+}\right)$(the symbol $\stackrel{D}{\rightarrow}$ means the weak convergence in $D\left(\mathbb{R}_{+}\right)$for the Skorokhod $J$-topology), where $(X(t))_{t \in \mathbb{R}_{+}}, X(0)=0$, is a nonnegative diffusion process with an infinitesimal operator $A$ such that

$$
A f(x)=\lambda f^{\prime}(x)+\frac{1}{2} \sigma^{2} x f^{\prime \prime}(x), \quad f \in C_{c}^{\infty}\left(\mathbb{R}_{+}\right) .
$$

Here $C_{c}^{\infty}\left(\mathbb{R}_{+}\right)$is the space of infinitely differentiable functions defined on $\mathbb{R}_{+}$and whose support is a compact set. The process $X$ is a (unique) solution of the following stochastic differential equation:

$$
d X(t)=\lambda d t+\sigma \sqrt{X(t)} d w(t), \quad t \in \mathbb{R}_{+},
$$

with the initial condition $X(0)=0$, where $(w(t))_{t \in \mathbb{R}_{+}}$is a standard Wiener process. Sriram [2] proved that

$$
\frac{1}{n} X^{(n)} \stackrel{D}{\rightarrow} X_{\alpha} \quad \text { as } n \rightarrow \infty,
$$

where $\left(X_{\alpha}(t)\right)_{t \in R_{+}}, X_{\alpha}(0)=0$, is a (nonnegative) diffusion process with an infinitesimal operator $A_{\alpha}$ such that

$$
A_{\alpha} f(x)=(\lambda+\alpha x) f^{\prime}(x)+\frac{1}{2} \sigma^{2} x f^{\prime \prime}(x), \quad f \in C_{c}^{\infty}\left(\mathbb{R}_{+}\right) .
$$

Sriram [2] assumed the following:

C1) $m_{n}=1+\alpha / n+o(1 / n)$ as $n \rightarrow \infty$ for some $\alpha \in \mathbb{R}$,

C2) $\sigma_{n}^{2} \rightarrow \sigma^{2}>0$ as $n \rightarrow \infty$,

C3) $\lambda_{n} \rightarrow \lambda>0$ and $b_{n}^{2} \rightarrow b^{2}>0$ as $n \rightarrow \infty$,

C4) for an arbitrary sequence $\left\{x_{n}\right\}$ such that $x_{n} \rightarrow x, 0<x<\infty$,

$$
\lim _{n \rightarrow \infty} \sigma_{n}^{-2} \mathrm{E}\left(\xi_{1,1}^{(n)}-m_{n}\right)^{2} I\left(\left|\xi_{1,1}^{(n)}-m_{n}\right|>\varepsilon \sigma_{n} \sqrt{n x_{n}}\right)=0
$$

for all $\varepsilon>0$.

The process $\left(X_{\alpha}(t)\right)_{t \in \mathbb{R}_{+}}$is a (unique) solution of the following stochastic differential equation:

$$
d X_{\alpha}(t)=\left(\lambda+\alpha X_{\alpha}(t)\right) d t+\sigma \sqrt{X_{\alpha}(t)} d w(t), \quad t \in \mathbb{R}_{+},
$$

with the initial condition $X_{\alpha}(0)=0$.

It is proved in $[3]$ that

$$
\frac{1}{n} X^{(n)} \stackrel{D}{\rightarrow} \mu \quad \text { as } n \rightarrow \infty
$$

where $\mu(t)=\lambda \int_{0}^{t} e^{\alpha s} d s, t \in \mathbb{R}_{+}$, if conditions C1) and C3) hold and if $\sigma_{n}^{2} \rightarrow 0$ as $n \rightarrow \infty$ (conditions such as $\mathrm{C} 4$ ) can be omitted in this case). It is clear that $\mu(t)$ is a solution of the following ordinary differential equation:

$$
d \mu(t)=(\lambda+\alpha \mu(t)) d t, \quad \mu(0)=0,
$$

and its operator $A_{\alpha}$ can be written as follows:

$$
A_{\alpha} f(x)=(\lambda+\alpha x) f^{\prime}(x), \quad f \in C_{c}^{\infty}\left(\mathbb{R}_{+}\right) .
$$

This result shows that the Sriram theorem mentioned above holds for the case where $\sigma_{n}^{2} \rightarrow 0$ (if one formally puts $\sigma=0$ in (2), then one obtains (44)).

The aim of this paper is to generalize the Sriram theorem for the case where the behavior $($ as $n \rightarrow \infty)$ of $m_{n}, \sigma_{n}^{2}$, and $\lambda_{n}, b_{n}^{2}$ differs from that in Theorem 3.1 of [2]. If 
$m_{n}=1+\alpha d_{n}^{-1}$ and $n d_{n}^{-1} \rightarrow 0$, then our result stated below implies that the process $X^{(n)}$ behaves as in the critical case where $m_{n}=1$, that is, $\alpha=0$.

Theorem. Let, for some $\gamma>0$,

A: $m_{n}=1+\alpha d_{n}^{-1}$ for $\alpha \in \mathbb{R}$, where the sequence of positive numbers $d_{n}$ is such that $n d_{n}^{-1} \rightarrow \beta<\infty$; we agree that $\beta=0$ if $\alpha=0$;

B: $n^{1-\gamma} \sigma_{n}^{2} \rightarrow \sigma^{2}>0$ as $n \rightarrow \infty$;

C: $n^{1-\gamma} \lambda_{n} \rightarrow \lambda \geq 0$ as $n \rightarrow \infty$;

D: 1) $n^{1-2 \gamma} b_{n}^{2} \rightarrow 0$ as $n \rightarrow \infty$ or 2) $n^{1-\gamma} b_{n}^{2} \rightarrow b^{2} \geq 0$ as $n \rightarrow \infty$;

F: $\sigma_{n}^{-2} \mathrm{E}\left(\left(\xi_{1,1}^{(n)}-m_{n}\right)^{2} I\left(\left|\xi_{1,1}^{(n)}-m_{n}\right|>\theta n^{\gamma / 2} \sigma_{n}\right)\right) \rightarrow 0$ as $n \rightarrow \infty$ for all $\theta>0$.

Then

$$
\frac{X^{(n)}}{n^{\gamma}} \stackrel{D}{\rightarrow} X_{\alpha, \beta} \quad \text { as } n \rightarrow \infty
$$

where $\left(X_{\alpha, \beta}(t)\right)_{t \in \mathbb{R}_{+}}$is a (nonnegative and unique) solution of the following stochastic differential equation:

$$
d X_{\alpha, \beta}(t)=\left(\lambda+\beta \alpha X_{\alpha, \beta}(t)\right) d t+\sigma \sqrt{X_{\alpha, \beta}(t)} d w(t), \quad X_{\alpha, \beta}(0)=0 .
$$

Note that Sriram's result follows from the latter theorem if $\beta=1, \gamma=1$, and if condition D2) holds.

As follows from results of [5. Chapter $4, \S 8]$ a solution of the latter equation is nonnegative and unique, indeed.

Proof of the theorem. We consider only the case where condition D1) is satisfied. It is not hard to see that $\left(X_{k}^{(n)} / n^{\gamma}, k=0,1, \ldots\right)$ is a Markov chain assuming values in the set $E_{n}=\left\{l / n^{\gamma}, l=0,1, \ldots\right\}$, since the families of random variables

$$
\left\{\xi_{k, j}^{(n)}, k, j \in \mathbb{N}\right\} \quad \text { and } \quad\left\{\varepsilon_{k}^{(n)}, k \in \mathbb{N}\right\}
$$

are independent. For all $f \in C_{c}^{\infty}\left(\mathbb{R}_{+}\right)$, define

$$
T_{n} f(x)=\mathrm{E} f\left\{n^{-\gamma}\left(\sum_{j=1}^{n^{\gamma} x} \xi_{1, j}^{(n)}+\varepsilon_{1}^{(n)}\right)\right\}, \quad x \in E_{n} .
$$

According to Theorem 6.5 of Chapter 1 and Corollary 8.9 of Chapter 4 in 4, we need to show that

$$
\lim _{n \rightarrow \infty} \sup _{x \in E_{n}}\left|\Delta_{n}^{f}(x)\right|=0
$$

for all $f \in C_{c}^{\infty}\left(\mathbb{R}_{+}\right)$, since $X^{(n)}(0)=0, n \in \mathbb{N}$, where

$$
\Delta_{n}^{f}(x)=n\left(T_{n} f(x)-f(x)\right)-(\lambda+\beta \alpha x) f^{\prime}(x)-\frac{1}{2} \sigma^{2} x f^{\prime \prime}(x) .
$$

Put

$$
S_{k}^{(n)}=\sum_{j=1}^{k}\left(\xi_{1, j}^{(n)}-1\right)+\varepsilon_{1}^{(n)}, \quad k, n \in \mathbb{N} .
$$

It is obvious that $T_{n} f(x)=\mathrm{E} f\left(x+n^{-\gamma} S_{n^{\gamma} x}^{(n)}\right)$. By the Taylor formula with the Schlömilch-Roche remainder term we get

$$
T_{n} f(x)=f(x)+f^{\prime}(x) n^{-\gamma} \mathrm{E} S_{n^{\gamma} x}^{(n)}+n^{-2 \gamma} \mathrm{E}\left(\left(S_{n^{\gamma} x}^{(n)}\right)^{2} \int_{0}^{1}(1-\nu) f^{\prime \prime}\left(x+\nu n^{-\gamma} S_{n^{\gamma} x}^{(n)}\right) d \nu\right) .
$$


For $x \in E_{n}$, we have

$$
\begin{gathered}
\mathrm{E} S_{n^{\gamma} x}^{(n)}=n^{\gamma}\left(m_{n}-1\right) x+\lambda_{n}, \\
\mathrm{E}\left(S_{n^{\gamma} x}^{(n)}\right)^{2}=n^{\gamma} \sigma_{n}^{2} x+b_{n}^{2}+\lambda_{n}^{2}+n^{2 \gamma}\left(m_{n}-1\right)^{2} x^{2}+2 n^{\gamma}\left(m_{n}-1\right) \lambda_{n} x .
\end{gathered}
$$

Now we represent $\Delta_{n}^{f}(x)$ in the following form:

$$
\Delta_{n}^{f}(x)=\Delta_{n, 1}^{f}(x)+\Delta_{n, 2}^{f}(x)+\Delta_{n, 3}^{f}(x),
$$

where

$$
\begin{gathered}
\Delta_{n, 1}^{f}(x)=f^{\prime}(x)\left(\left(n\left(m_{n}-1\right)-\beta \alpha\right) x+n^{1-\gamma} \lambda_{n}-\lambda\right), \\
\Delta_{n, 2}^{f}(x)=n^{1-2 \gamma} \mathrm{E}\left(\left(S_{n^{\gamma} x}^{(n)}\right)^{2} \int_{0}^{1}(1-\nu)\left(f^{\prime \prime}\left(x+\nu n^{-\gamma} S_{n^{\gamma} x}^{(n)}\right)-f^{\prime \prime}(x)\right) d \nu\right), \\
\Delta_{n, 3}^{f}(x)=\frac{1}{2}\left[\left(n^{1-\gamma} \sigma_{n}^{2}-\sigma^{2}\right) x+n^{1-2 \gamma}\left(b_{n}^{2}+\lambda_{n}^{2}\right)\right. \\
\left.\quad+n\left(m_{n}-1\right)^{2} x^{2}+2\left(m_{n}-1\right) n^{1-\gamma} \lambda_{n} x\right] f^{\prime \prime}(x) .
\end{gathered}
$$

To prove equality (5), one needs to show that

$$
\lim _{n \rightarrow \infty} \Delta_{n}^{f}\left(x_{n}\right)=0
$$

for any sequence $\left\{x_{n}\right\}$ such that $x_{n} \in E_{n}, n \in \mathbb{N}$, and $x_{n} \rightarrow x \in[0, \infty]$ as $n \rightarrow \infty$. Assume that the support of the function $f$ coincides with the interval $[0, c]$. According to conditions $\mathrm{A}$ and $\mathrm{C}$ we get

$$
\Delta_{n, 1}^{f}\left(x_{n}\right)=f^{\prime}\left(x_{n}\right)\left(\left(\alpha n d_{n}^{-1}-\beta \alpha\right) x_{n}+n^{1-\gamma} \lambda_{n}-\lambda\right) \rightarrow 0
$$

as $n \rightarrow \infty$ if $x_{n} \rightarrow x \in[0, \infty]$.

Conditions A-D imply that

$$
\begin{aligned}
\Delta_{n, 3}^{f}\left(x_{n}\right)=\frac{1}{2}\left[\left(n^{1-\gamma} \sigma_{n}^{2}-\sigma^{2}\right) x_{n}\right. & +n^{1-2 \gamma} b_{n}^{2}+\frac{\left(n^{1-\gamma} \lambda_{n}\right)^{2}}{n} \\
& \left.+\frac{\left(\alpha n d_{n}^{-1}\right)^{2}}{n} x_{n}^{2}+2 n^{1-\gamma} \lambda_{n} \alpha d_{n}^{-1} x_{n}\right] f^{\prime \prime}\left(x_{n}\right) \rightarrow 0
\end{aligned}
$$

as $n \rightarrow \infty$ if $x_{n} \rightarrow x \in[0, \infty]$.

Now we estimate $\Delta_{n, 2}^{f}\left(x_{n}\right)$. It is obvious that

$$
x+\nu n^{-\gamma} S_{n^{\gamma} x}^{(n)} \geq x(1-\nu) .
$$

If $\nu<1-c / x$, then $x(1-\nu) \geq c$, whence

$$
f^{\prime \prime}\left(x+\nu n^{-\gamma} S_{n^{\gamma} x}^{(n)}\right) \equiv 0
$$

for $\nu<1-c / x$. Further

$$
\begin{aligned}
n^{1-2 \gamma} & \left(S_{n^{\gamma} x}^{(n)}\right)^{2} \int_{0}^{1}(1-\nu)\left|f^{\prime \prime}\left(x+\nu n^{-\gamma} S_{n^{\gamma} x}^{(n)}\right)-f^{\prime \prime}(x)\right| d \nu \\
& \leq n^{1-2 \gamma}\left(S_{n^{\gamma} x}^{(n)}\right)^{2}\left[\left|f^{\prime \prime}(x)\right| \int_{0}^{0 \vee\left(1-\frac{c}{x}\right)}(1-\nu) d \nu+2\left\|f^{\prime \prime}\right\| \int_{0 \vee\left(1-\frac{c}{x}\right)}^{1}(1-\nu) d \nu\right] \\
& \leq n^{1-2 \gamma}\left(S_{n^{\gamma} x}^{(n)}\right)^{2}\left[\left|f^{\prime \prime}(x)\right|+\left\|f^{\prime \prime}\right\|\left(\left(\frac{c}{x}\right)^{2} \wedge 1\right)\right]
\end{aligned}
$$

Therefore

$$
\left|\Delta_{n, 2}^{f}(x)\right| \leq n^{1-2 \gamma} \mathrm{E}\left(S_{n^{\gamma} x}^{(n)}\right)^{2}\left[\left|f^{\prime \prime}(x)\right|+\left\|f^{\prime \prime}\right\|\left(\left(\frac{c}{x}\right)^{2} \wedge 1\right)\right] .
$$


If $x_{n} \rightarrow 0$, then the latter relation together with (7) implies that

$$
\begin{aligned}
\lim _{n \rightarrow \infty}\left|\Delta_{n, 2}^{f}\left(x_{n}\right)\right| \leq & \lim _{n \rightarrow \infty}\left[n^{1-\gamma} \sigma_{n}^{2} x_{n}+\right. \\
+ & n^{1-2 \gamma} b_{n}^{2}+\frac{\left(n^{1-\gamma} \lambda_{n}\right)^{2}}{n} \\
& \left.+\frac{\left(\alpha n d_{n}^{-1}\right)^{2} x_{n}^{2}}{n}+2 n^{1-\gamma} \lambda_{n} \alpha d_{n}^{-1} x_{n}\right] \\
& \times\left(\left|f^{\prime \prime}\left(x_{n}\right)\right|+\left\|f^{\prime \prime}\right\|\left(\left(\frac{c}{x_{n}}\right)^{2} \wedge 1\right)\right)=0
\end{aligned}
$$

by the assumptions of the theorem.

Consider the case of $x_{n} \rightarrow \infty$ as $n \rightarrow \infty$. In this case,

$$
\mathrm{E}\left(\frac{S_{n^{\gamma} x_{n}}^{(n)}}{\sigma_{n} \sqrt{n^{\gamma} x_{n}}}\right)^{2} \rightarrow 1
$$

Then we obtain from (13) that

$$
\begin{aligned}
\lim _{n \rightarrow \infty} & \left|\Delta_{n, 2}^{f}\left(x_{n}\right)\right| \\
& \leq \lim _{n \rightarrow \infty} n^{1-\gamma} \sigma_{n}^{2} x_{n} \mathrm{E}\left(\frac{S_{n^{\gamma} x_{n}}^{(n)}}{\sigma_{n} \sqrt{n^{\gamma} x_{n}}}\right)^{2}\left(\left|f^{\prime \prime}\left(x_{n}\right)\right|+\left\|f^{\prime \prime}\right\|\left(\left(\frac{c}{x_{n}}\right)^{2} \wedge 1\right)\right) \\
& =\sigma^{2}\left\|f^{\prime \prime}\right\| \lim _{n \rightarrow \infty} x_{n}\left(\left(\frac{c}{x_{n}}\right)^{2} \wedge 1\right)=0 .
\end{aligned}
$$

It remains to consider the case where $x_{n} \rightarrow x$ as $n \rightarrow \infty$ for some $0<x<\infty$.

By conditions B and C,

$$
\mathrm{E}\left(\frac{\varepsilon_{1}^{(n)}}{\sigma_{n} \sqrt{n^{\gamma} x_{n}}}\right)=\frac{n^{1-\gamma} \lambda_{n}}{\sigma_{n} n^{(1-\gamma) / 2} x_{n}} \cdot \frac{1}{\sqrt{n}} \rightarrow 0 \quad \text { as } n \rightarrow \infty
$$

Then the Chebyshev inequality implies that

It is obvious that

$$
\frac{\varepsilon_{1}^{(n)}}{\sigma_{n} \sqrt{n^{\gamma} x_{n}}} \stackrel{\mathrm{P}}{\rightarrow} 0 \quad \text { as } n \rightarrow \infty .
$$

$$
\frac{1}{\sigma_{n} \sqrt{n^{\gamma} x_{n}}}\left|\sum_{j=1}^{n^{\gamma} x_{n}}\left(\xi_{1, j}^{(n)}-m_{n}\right)-\sum_{j=1}^{n^{\gamma} x_{n}}\left(\xi_{1, j}^{(n)}-1\right)\right| \leq \frac{n^{\gamma} x_{n} \alpha}{d_{n} \sigma_{n} \sqrt{n^{\gamma} x_{n}}} \sim \frac{\sqrt{x_{n}} \alpha n^{1 / 2}}{\left(n^{1-\gamma} \sigma_{n}^{2}\right)^{1 / 2} d_{n}} \rightarrow 0
$$

as $n \rightarrow \infty$. This result, condition $\mathrm{F}$, and the central limit theorem imply that

$$
\frac{S_{n^{\gamma} x_{n}}^{(n)}}{\sigma_{n} \sqrt{n^{\gamma} x_{n}}} \Rightarrow N \quad \text { as } n \rightarrow \infty,
$$

where $N$ is a random variable whose distribution is normal with parameters $(0,1)$ and where the symbol $\Rightarrow$ denotes the weak convergence. Taking into account condition B of the theorem we conclude that

$$
n^{1-2 \gamma}\left(S_{n^{\gamma} x_{n}}^{(n)}\right)^{2}=n^{1-\gamma} \sigma_{n}^{2} x_{n} \cdot\left(\frac{S_{n \gamma}^{(n)} x_{n}}{\sigma_{n} \sqrt{n^{\gamma} x_{n}}}\right)^{2} \Rightarrow \sigma^{2} x N^{2} \quad \text { as } n \rightarrow \infty .
$$

Further,

$$
\left|\int_{0}^{1}(1-v)\left(f^{\prime \prime}\left(x_{n}+v n^{-\gamma} S_{n^{\gamma} x_{n}}\right)-f^{\prime \prime}\left(x_{n}\right)\right) d v\right| \leq\left\|f^{\prime \prime \prime}\right\| n^{-\gamma}\left|S_{n^{\gamma} x_{n}}^{(n)}\right| .
$$


Since

we get

$$
\begin{gathered}
\mathrm{E} n^{-\gamma} S_{n^{\gamma} x_{n}}^{(n)}=\alpha d_{n}^{-1} x_{n}+\frac{n^{1-\gamma} \lambda_{n}}{n} \rightarrow 0, \quad n \rightarrow \infty, \\
\operatorname{Var}\left(n^{-\gamma} S_{n^{\gamma} x_{n}}^{(n)}\right)=\frac{n^{1-\gamma} \sigma_{n}^{2} x_{n}}{n}+\frac{b_{n}^{2} n^{1-2 \gamma}}{n} \rightarrow 0, \quad n \rightarrow \infty,
\end{gathered}
$$

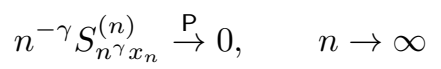

(this also follows from the central limit theorem (16)).

Hence relations (16) -(18) imply that the random variable under the sign of the mathematical expectation in the expression $\Delta_{n, 2}^{f}\left(x_{n}\right)$ approaches zero in probability.

Applying (16)-(17) we conclude that the right hand side of inequality (12) weakly converges to

Since

$$
\sigma^{2} x\left(\left|f^{\prime \prime}(x)\right|+\left\|f^{\prime \prime}\right\|\left(\left(\frac{c}{x}\right)^{2} \wedge 1\right)\right) N^{2} .
$$

$$
\begin{gathered}
\lim _{n \rightarrow \infty} n^{1-2 \gamma}\left(\left|f^{\prime \prime}\left(x_{n}\right)\right|+\left\|f^{\prime \prime}\right\|\left(\left(\frac{c}{x_{n}}\right)^{2} \wedge 1\right)\right) \mathrm{E}\left(S_{n \gamma}^{(n)} x_{n}\right)^{2} \\
=\mathrm{E}\left[\sigma^{2} x\left(\left|f^{\prime \prime}(x)\right|+\left\|f^{\prime \prime}\right\|\left(\left(\frac{c}{x}\right)^{2} \wedge 1\right)\right) N^{2}\right]<\infty,
\end{gathered}
$$

we obtain

$$
\lim _{n \rightarrow \infty} \Delta_{n, 2}^{f}\left(x_{n}\right)=0
$$

by the dominated convergence theorem 4 .

This together with relations (14), (15), (10), and (11) yields (9) and thus completes the proof of the theorem.

Example. Let $0<\tau<\frac{1}{2}, \delta \geq 1$, and let the random variable $\xi_{1,1}^{(n)}$ assume the values 0 , 1 , and 2 with the probabilities $n^{-\tau}-n^{-\delta}, 1-2 n^{-\tau}+n^{-\delta}$, and $n^{-\tau}$, respectively. It is not complicated to see that

$$
m_{n}=1+n^{-\delta}, \quad \sigma_{n}^{2}=2 n^{-\tau}-n^{-\delta}-n^{-2 \delta}=2 n^{-\tau}+o\left(n^{-\tau}\right) .
$$

Thus conditions A and B of the theorem hold with $\alpha=1, d_{n}=n^{\delta}, \gamma=1-\tau$, and $\sigma^{2}=2$.

Let the immigration $\varepsilon_{k}^{(n)}$ have the Poisson distribution with parameter $\lambda_{n}$ and let there be a number $\lambda>0$ such that

$$
n^{\tau} \lambda_{n} \rightarrow \lambda \text { as } n \rightarrow \infty \text {. }
$$

Thus conditions $\mathrm{C}$ and D of the theorem hold, too. Further

$$
n^{\gamma / 2} \sigma_{n}=\sqrt{2} n^{(1-2 \tau) / 2}+o(1) \rightarrow \infty \quad \text { as } n \rightarrow \infty .
$$

Then, for all sufficiently large $n$ and for all $\theta>0$,

$$
I\left(\left|\xi_{1,1}^{(n)}-m_{n}\right|>\theta n^{\gamma / 2} \sigma_{n}\right)=0
$$

almost surely. This implies condition $\mathrm{F}$ of the theorem.

In the case under consideration, the limit process $X_{\beta}$ is a solution of the stochastic differential equation

$$
d X_{\beta}(t)=\left(\lambda+\beta X_{\beta}(t)\right) d t+\sqrt{2 X_{\beta}(t)} d W(t), \quad X(0)=0,
$$

where $\beta=1$ if $\delta=1$, and $\beta=0$ if $\delta>1$. 


\section{BIBLIOGRAPHY}

1. C. Z. Wei and J. Winnicki, Some asymptotic results for the branching process with immigration, Stoch. Process. Appl. 31 (1989), 261-282. MR998117 (91c:60109)

2. T. N. Sriram, Invalidity of bootstrap for critical branching process with immigration, Ann. Statist. 22 (1994), 1013-1023. MR.1292554 (95g:62098)

3. M. Ispany, G. Pap, and M. C. A. Van Zuijlen, Fluctuation limit of branching processes with immigration and estimation of the means, Adv. Appl. Probab. 37 (2005), 523-538. MR2144565 (2006c:60103)

4. S. N. Ethier and T. G. Kurtz, Markov Processes, John Wiley, New York, 1986. MR838085 (88a:60130)

5. N. Ikeda and S. Watanabe, Stochastic Differential Equations and Diffusion Processes, NorthHolland Publishing Co., Kodansha, Ltd., Amsterdam-New York-Tokyo, 1981. MR637061 (84b:60080)

Department of Probability Theory and Mathematical Statistics, Institute of Mathematics and Information Technologies, F. Khodzhaev Street 29, Tashkent 100125, Uzbekistan

E-mail address: yakubjank@mail.ru

Received 6/AUG/2007

Translated by OLEG KLESOV 